\title{
Dominant Frequency Variability Mapping for Identifying Stable Drivers During Persistent Atrial Fibrillation Using Non-Contact Mapping
}

\author{
Xin $\mathrm{Li}^{1}$, Gavin S Chu ${ }^{1,2}$, Tiago P Almeida ${ }^{5}$, João L Salinet ${ }^{6}$, Amar R Mistry ${ }^{1}$, Zakariyya Vali ${ }^{1}$, \\ Peter J Stafford ${ }^{2}$, Fernando S Schlindwein ${ }^{3,4}$, G André $\mathrm{Ng}^{1,2,3}$ \\ ${ }^{1}$ Department of Cardiovascular Sciences, University of Leicester, Leicester, UK \\ ${ }^{2}$ University Hospitals of Leicester NHS Trust, UK \\ ${ }^{3}$ National Institute for Health Research Leicester Cardiovascular Biomedical Research Centre, UK \\ ${ }^{4}$ Department of Engineering, University of Leicester, Leicester, UK \\ ${ }^{5}$ Aeronautics Institute of Technology, ITA, Brazil \\ ${ }^{6}$ Biomedical Engineering, Centre for Engineering, Modelling and Applied Social Sciences, Federal \\ ABC University, Brazil
}

\begin{abstract}
Catheter ablation is a widely-used therapy to treat atrial fibrillation $(A F)$, but the identification of ablation targets remain challenging in persistent $A F$ (persAF). Dominant frequency $(D F)$ mapping has been shown to be spatiotemporally unstable in persAF, with $D F$ variability $(D F V)$ correlating with the spectral organisation index (OI). This study aims to assess DFV at ablation sites between patients with $A F$ termination and non-termination.

10 persAF patients undergoing LA ablation were enrolled. AF was terminated in 4 patients after ablating highest DFs. 2048-channel virtual electrograms (Ensite Array) were analysed in Matlab. DFV index (DFVI) was proposed to quantify DF temporal stability. Mock ablation targets were identified based on DFVI and the percentage of region actually ablated was computed.

Ablation sites in termination patients revealed higher OI and lower DFVI. In the termination group, a greater proportion of DFVI was ablated. Atrial regions with higher temporal stability and organisation may offer more precise locations of stable focal drivers and may lead to higher success in AF termination following ablation.
\end{abstract}

\section{Introduction}

Atrial fibrillation (AF) is one of the most common cardiac arrhythmias in clinical practice, affecting 1-2\% of the general population [1]. The loss of effective atrial contraction in AF patients can result in blood clots in the atria, which increases the risk of stroke five-fold [2]. It is generally accepted that $\mathrm{AF}$ requires a trigger for its initiation and a substrate for the perpetuation [3]. Catheter ablation is a widely-used therapy and treats AF by either eliminating the trigger or by altering the arrhythmogenic substrate [4]. Treating paroxysmal AF (pAF) with pulmonary veins isolation (PVI) in clinical practice is based on evidence that triggered firing originates from PVs [5,6], but the poor results in treating persistent AF (PersAF) patients points to the importance of AF drivers outside the pulmonary veins [7], with the underlying atrial substrate being either structural or electrical [8]. Human atrial sites hosting high dominant frequency (DF) has shown to play an important role for the maintenance of AF [9]. However, part of these regions can be spatiotemporally unstable [10], making them difficult to target. On the other hand, some believe temporally stable DF has the potential to represent relevant and stable focal drivers [11]. Panoramic mapping using electrogram (EGM) recordings provides excellent simultaneous data for assessing frequency features such as DF and organisation index (OI) and their temporal stability and variation. Although, DF variability (DFV) has been shown to have a certain degree of correlation with OI [12], it has not been used as predictive marker for identifying targets for catheter ablation during persAF. In this study, we aim to investigate the potential use of DFV as an individual marker by comparing ablation sites between patients who had termination of persAF against those without termination.

\section{Materials and Methods}

\subsection{Data Acquisition}

Ten persAF patients undergoing first time left atrial (LA) catheter ablation were enrolled. 30s of LA noncontact electrograms (EGMs, Ensite Array, St Jude Med- 
ical) were exported to our Matlab platform [13] to guide ablation targeting DF. High DF regions in the LA were identified as previously described [10]. Four out of ten patients had AF terminations (3 flutter, 1 sinus rhythm) by high DF ablation before pulmonary veins isolation (PVI) [14]. There were no adverse events in any of our ten patients.

\subsection{Signal Pre-processing}

The EGMs were sampled at $2034.5 \mathrm{~Hz}$ and then resampled to $512 \mathrm{~Hz}$, and 10 seconds of the 2048-channel EGMs were analysed per patient. Subtraction of the QRS, as described in previous work [15], was performed to prevent ventricular farfield sensing causing misleading frequency components.

\subsection{Dominant Frequency}

Spectral analysis was carried out by performing shorttime Fourier transform (STFT). The EGMs were then divided into 2 seconds sliding windows with a $95 \%$ overlap with 0.1 second as sliding step. The discrete form of STFT is expressed as Equation 1.

$$
\operatorname{STFT}\{x[n]\}(m, \omega)=\sum_{n=-\infty}^{\infty} x[n] w[n-m] e^{-j \omega n}
$$

$x[n]$ is the time series signal, $m$ is the timing of sliding window and $w[n]$ is the windowing function. A Hamming window was used to reduce the amplitude of the side lobes around the DF peak in the power spectrum. This setting of a 2-second window with a large overlap can benefit from sensing small temporal changes, but also maintaining acceptable frequency resolution with $0.5 \mathrm{~Hz}$ as a unit step. A zero padding factor of 5 was applied when performing the FFT, resulting in a frequency step of $0.1 \mathrm{~Hz}$. DF was defined as the frequency peak in the power spectrum within the physiological range of $4-10 \mathrm{~Hz}$ in each sliding window. OI is defined as the ratio of the area of the DF peak $( \pm 0.25$ $\mathrm{Hz}$ ) together with its harmonics, and the total area of the power spectrum (up to $20 \mathrm{~Hz}$ ).

\subsection{DF Variability Index}

With the hypothesis to target stable sources, DFV index (DFVI) was proposed to quantify DF temporal stability. Firstly, the DF standard deviation $\left(D F_{s d}\right)$ of each channel/node was calculated for each patient according to Equation 2, generating a single $D F_{s d}$ map with 2048 nodes. DFVI was then calculated based on the $D F_{s d}$ map, and normalised against the maximum variance per patient (Equation 3), ranging from 0 to 1 (a.u. arbitrary units).

$$
\begin{gathered}
D F_{s d}[i]=\sqrt{\frac{\sum_{j=1}^{N}(D F[i, j]-\overline{D F[i,:]})^{2}}{N-1}} \\
D F V I[i]=\frac{D F_{s d}[i]}{\max _{i \in[1,2048]}\left(D F_{s d}[i]\right)}
\end{gathered}
$$

$i$ is the channel/node index, while $j$ is the window number and $N$ is the total number of windows. The bar denotes the average value and colon is used for selecting all columns.

\subsection{Mock ablation targets}

With the aim to assess the effectiveness of the DFVI markers, mock ablation targets were pre-defined as the atrial regions with DFVI below 0.15 (a.u.) for each patient. The percentage of mock targets that have been truly ablated during the procedure were calculated for each patient.

\subsection{Statistical Analysis}

All continuous normally distributed variables are expressed as mean \pm standard deviation (SD), and the non normal distributed variables are expressed as median and interquartile range (IQR). Unpaired t tests were applied for normally distributed variables and KolmogorovSmirnov test was used for non-parametric features. P-values of less than 0.05 were considered statistically significant.

\section{Results}

A total number of 1500 lesion nodes in termination patient $(\mathrm{N}=4)$ and 1970 in non-termination patients $(\mathrm{N}=6)$ were ablated. Figure 1 A summaries the outcome of the DFVI approach, the DFVI maps showed less overlap between the low DFVI area (red) and the atrial regions that were truly ablated (green dots) in the nontermination patients than the termination group. The DF values within the ablation lesions were lower in termination than non-termination $(5.54 \pm 0.32$ vs $6.19 \pm 0.41 \mathrm{~Hz}$, $\mathrm{p}<0.0001$, Figure $1 \mathrm{~B}$ (i)). The OIs in ablation lesions were slightly higher in termination $(0.48 \pm 0.07$ vs 0.45 $\pm 0.07, \mathrm{p}<0.0001$, Figure $1 \mathrm{~B}$ (ii)). Moreover, DFVIs of the ablated atrial regions were significantly lower in termination patients than the non-termination patients $(0.25$ \pm 0.21 vs $0.45 \pm 0.20, \mathrm{p}<0.0001$, Figure $1 \mathrm{~B}$ (iii)). The percentage of regions defined as mock targets that were ablated was higher in termination 17.9\% [IQR 15.0 - $27.4 \%$ ] than in non-termination 8.2\% [IQR 3.9-14.0\%] ( $\mathrm{p}=0.04$, Figure 1 B (iv)). 


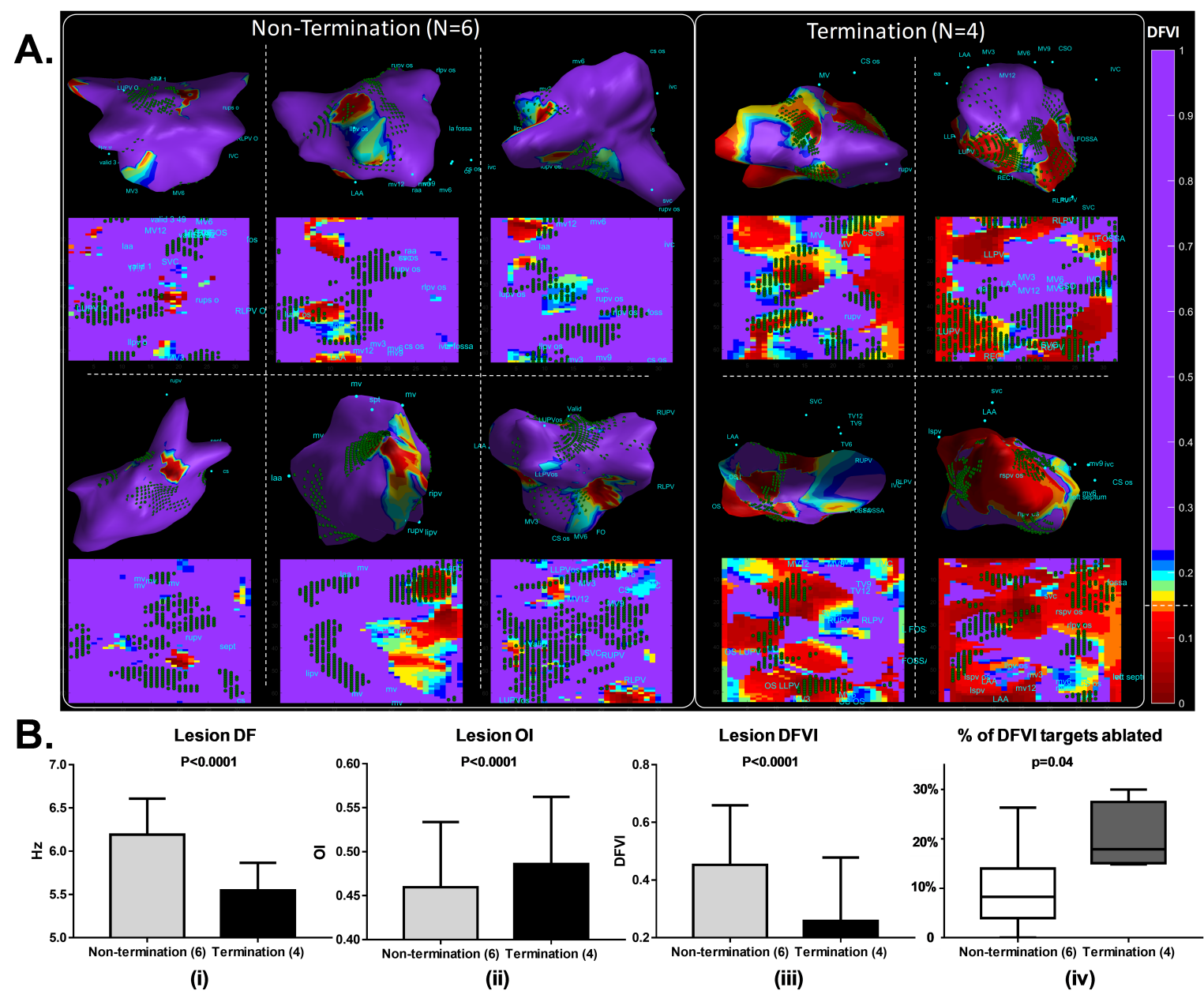

Figure 1. A. DFVI maps (both 3D and 2D) of all ten patients reconstructed in Matlab. The green dots represent the ablation lesions marked by the mapping system. A higher percentage of DFVI targets were ablated in the termination group compared to non-termination group; B. (i) the DFs of ablation lesion in terminated patients and non-terminated patients (mean \pm SD) (ii) the OIs of ablation lesion in terminated patients and non-terminated patients (mean \pm SD) (iii) the DFVIs of ablation lesion in terminated patients and non-terminated patients (mean \pm SD) (iv) box and whisker plots (box: IQR, bars: min-max) of the percentage of mock ablation targets defined by DFVI smaller than 0.15 (a.u.) in termination patients and non-termination patients.

\section{Discussions}

From our results it can be observed that lower DFs were found in ablation lesion from termination patients, which is an interesting result, as highest DFs were targeted within patients. This could be firstly explained by the slower overall atrial rates in the termination group, but cycle length data suggested this difference was not significant from 10 patients. Further investigation is needed to study the effect of baseline atrial rate and ablation success rate in persAF patients. The DFVIs, however, being unrelated to overall $\mathrm{DF}$ values or atrial rates, were found to be significantly lower in termination group. Also, a lager percentage of the DFVI targets were ablated in termination patients from this small cohort. These findings may indicate the important potential of DFVI. DF variance has shown to correlate with OI previously [12], and increase in OI was shown to predict AF termination [16]. As mentioned, high DF regions were believed to be the ablation targets $[9,17]$, but their spatio-temporal instability makes it challenging to summarise the behaviour using short EGM recordings[10]. As such, the advantages of using the simple DFVI are: 1) being normalised on different patients' variation scales to a notionally common scale and not related to atrial rate; 2) being able to be applied to a short segment with clear mathematical expression, that is suitable for use in a electrophysiological clinical environment. 


\section{Conclusions}

We have proposed and used DF variability as a marker for identifying ablation targets in persAF. Ablation sites in termination patients revealed higher OI and lower DFVI. A greater percentage of DFVI targets were ablated in the termination group. Atrial regions with higher temporal stability and organisation may offer more precise locations of stable focal drivers and may lead to higher success in AF termination following ablation. DFVI values were derived and processed from a segment duration of only $10 \mathrm{sec}-$ onds when increasing the overlap between time windows. Further study on the optimal duration to maintain acceptable DF temperate stability using longer time windows is under-way. The technique allows for real time implementation into our software tool [13] to guide ablation in future clinical studies.

\section{Acknowledgements}

This work was supported by the NIHR Leicester Biomedical Research Centre. XL was funded by Medical Research Council, UK. TPA received research grants from CAPES and FAPESP (n. 2017/00319-8).

\section{References}

[1] Lip GY, Fauchier L, Freedman SB, Van Gelder I, Natale A, Gianni C, Nattel S, Potpara T, Rienstra M, Tse HF, Lane DA. Atrial fibrillation. Nat Rev Dis Primers 2016;2:16016.

[2] Oral H. Atrial Fibrillation: Mechanisms , Features , and Management Pathophysiology, volume Vol. 2000. 5th ed. edition. Philadelphia, Pa.: Saunders, 2009; 119125.

[3] Nattel S. New ideas about atrial fibrillation 50 years on. Nature 2002;415(6868):219-26.

[4] Haissaguerre M, Marcus FI, Fischer B, Clementy J. Radiofrequency catheter ablation in unusual mechanisms of atrial fibrillation: report of three cases. J Cardiovasc Electrophysiol 1994;5(9):743-51.

[5] Jais P, Haissaguerre M, Shah DC, Chouairi S, Gencel L, Hocini M, Clementy J. A focal source of atrial fibrillation treated by discrete radiofrequency ablation. Circulation 1997;95(3):572-6.

[6] Haissaguerre M, Jais P, Shah DC, Takahashi A, Hocini M, Quiniou G, Garrigue S, Le Mouroux A, Le Metayer P, Clementy J. Spontaneous initiation of atrial fibrillation by ectopic beats originating in the pulmonary veins. N Engl J Med 1998;339(10):659-66.

[7] Jalife J, Berenfeld O, Mansour M. Mother rotors and fibrillatory conduction: a mechanism of atrial fibrillation. Cardiovasc Res 2002;54(2):204-16.

[8] Morillo CA, Klein GJ, Jones DL, Guiraudon CM. Chronic rapid atrial pacing. structural, functional, and electrophysiological characteristics of a new model of sustained atrial fibrillation. Circulation 1995;91(5):1588-95.

[9] Sanders P, Berenfeld O, Hocini M, Jais P, Vaidyanathan
R, Hsu LF, Garrigue S, Takahashi Y, Rotter M, Sacher F, Scavee C, Ploutz-Snyder R, Jalife J, Haissaguerre M. Spectral analysis identifies sites of high-frequency activity maintaining atrial fibrillation in humans. Circulation 2005; 112(6):789-97.

[10] Salinet JL, Tuan JH, Sandilands AJ, Stafford PJ, Schlindwein FS, Ng GA. Distinctive patterns of dominant frequency trajectory behavior in drug-refractory persistent atrial fibrillation: preliminary characterization of spatiotemporal instability. J Cardiovasc Electrophysiol 2014;25(4):371-379.

[11] Kimata A, Yokoyama Y, Aita S, Nakamura H, Higuchi K, Tanaka Y, Nogami A, Hirao K, Aonuma K. Temporally stable frequency mapping using continuous wavelet transform analysis in patients with persistent atrial fibrillation. $\mathrm{J}$ Cardiovasc Electrophysiol 2018;

[12] Jarman JWE, Wong T, Kojodjojo P, Spohr H, Davies JER, Roughton M, Francis DP, Kanagaratnam P, O'Neill MD, Markides V, Davies DW, Peters NS. Organizational index mapping to identify focal sources during persistent atrial fibrillation. J Cardiovasc Electrophysiol 2014;25(4):355363.

[13] Li X, Salinet JL, Almeida TP, Vanheusden FJ, Chu GS, $\mathrm{Ng} \mathrm{GA}$, Schlindwein FS. An interactive platform to guide catheter ablation in human persistent atrial fibrillation using dominant frequency, organization and phase mapping. Comput Methods Programs Biomed 2017;141:83-92.

[14] Chu G, Li X, Vanheusden F, Almeida T, Salinet J, Dastagir N, Varanasi S, Chin S, Siddiqui S, Man S, Stafford P, Sandilands A, Schlindwein F, Ng G. Targeting cyclical highest dominant frequency in the ablation of persistent atrial fibrillation. Europace 2016;17(suppl 5):v1-v2.

[15] Salinet J. L. J, Madeiro JP, Cortez PC, Stafford PJ, Ng GA, Schlindwein FS. Analysis of QRS-T subtraction in unipolar atrial fibrillation electrograms. Med Biol Eng Comput 2013;51(12):1381-91.

[16] Tuan J, Osman F, Jeilan M, Kundu S, Mantravadi R, Stafford PJ, Ng GA. Increase in organization index predicts atrial fibrillation termination with flecainide post-ablation: spectral analysis of intracardiac electrograms. Europace 2010;12(4):488-93.

[17] Mansour M, Mandapati R, Berenfeld O, Chen J, Samie FH, Jalife J. Left-to-right gradient of atrial frequencies during acute atrial fibrillation in the isolated sheep heart. Circulation 2001;103(21):2631-6.

Address for correspondence:

Dr. Xin Li

Department of Cardiovascular Sciences

University of Leicester, UK

x1251@1e.ac.uk 